\title{
A System-Based Intervention to Improve Access to Hyperacute Stroke Care
}

\author{
Richard H. Swartz, Elizabeth Linkewich, Shelley Sharp, Jacqueline Willems, \\ Chris Olynyk, Nicola Tahair, Megan L. Cayley, Mark T. Bayley
}

\begin{abstract}
Background: Hyperacute stroke is a time-sensitive emergency for which outcomes improve with faster treatment. When stroke systems are accessed via emergency medical services (EMS), patients are routed to hyperacute stroke centres and are treated faster. But over a third of patients with strokes do not come to the hospital by EMS, and may inadvertently arrive at centres that do not provide acute stroke services. We developed and studied the impact of protocols to quickly identify and move "walk-in" patients from non-hyperacute hospitals to regional stroke centres (RSCs). Methods and Results: Protocols were developed by a multi-disciplinary and multi-institutional working group and implemented across 14 acute hospital sites within the Greater Toronto Area in December of 2012. Key metrics were recorded 18 months pre- and post-implementation. The teams regularly reviewed incident reports of protocol non-adherence and patient flow data. Transports increased by $80 \%$ from 103 to 185 . The number of patients receiving tissue plasminogen activator (tPA) increased by $68 \%$ from 34 to 57. Total EMS transport time decreased 17 minutes (mean time of 54.46 to 37.86 minutes, $p<0.0001$ ). Calls responded to within 9 minutes increased from 34 to 59\%. Conclusions: A systems-based approach that included a multi-organizational collaboration and consensus-based protocols to move patients from non-hyperacute hospitals to RSCs resulted in more patients receiving hyperacute stroke interventions and improvements in EMS response and transport times. As hyperacute stroke care becomes more centralized and endovascular therapy becomes more broadly implemented, the protocols developed here can be employed by other regions organizing patient flow across systems of stroke care.
\end{abstract}

RÉSUMÉ: Une intervention systémique pour améliorer l'accès aux soins pour les patients atteints d'un accident vasculaire cérébral suraigu. Contexte: L'accident vasculaire cérébral (AVC) suraigu nécessite un traitement d'urgence dont les résultats sont d'autant meilleurs que le traitement est administré plus rapidement. Le facteur temps est donc critique. Quand l'accès aux systèmes de prévention de l'AVC se fait par les services médicaux d'urgence (SMU), les patients sont dirigés vers des centres de traitement de l'AVC suraigu et sont traités plus rapidement. Cependant, plus du tiers des patients atteints d'un AVC ne sont pas conduits à l'hôpital par les SMU et peuvent être dirigés par inadvertance vers des centres qui ne fournissent pas de tels soins. Nous avons élaboré et étudié l'impact de protocoles pour identifier et transporter rapidement les patients qui se présentent à un centre où des soins suraigus ne sont pas offerts vers un centre régional de traitement de l'AVC. Méthodologie et résultats: Un groupe de travail multidisciplinaire et multi-institutionnel a élaboré des protocoles qui ont été déployés dans 14 hôpitaux de soins aigus dans la grande région de Toronto en décembre 2012. Les indicateurs clés ont été comptabilisés 18 mois avant et 18 mois après leur mise en œuvre. Les équipes ont revu régulièrement les rapports d'incidents de non-observance du protocole et les données de cheminement des patients. Les transports ont augmenté de $80 \%$, soit de 103 à 185 . Le nombre de patients qui ont reçu de l'activateur du plasminogène tissulaire (APt) a augmenté de $68 \%$, soit de 34 à 57 patients. Le temps de transport par SMU a diminué de 17 minutes (le temps moyen de 54,46 est passé à 37,86 minutes, p < 0,0001). Les réponses en dedans de 9 minutes ou moins ont augmenté de 34 à 59\%. Conclusions: Une approche systémique, qui incluait une collaboration multi-organisationnelle et des protocoles fondés sur un consensus de transport des patients d'un hôpital où les soins de l'AVC suraigu ne sont pas disponibles vers un centre régional de traitement de l'AVC, s'est traduite par une augmentation du nombre des patients qui reçoivent des interventions pour un AVC suraigu et par des améliorations de la réponse des SMU et des temps de transport. Comme les soins de l'AVC suraigu deviennent plus centralisés et le traitement endovasculaire est plus largement utilisé, les protocoles élaborés ici peuvent être appliqués dans d'autres régions pour organiser le transport des patients vers les centres de soins de l'AVC.

Keywords: Stroke, stroke care, acute stroke, thrombolysis (tPA)

doi:10.1017/cjn.2017.40

Can J Neurol Sci. 2017; 44: 475-482

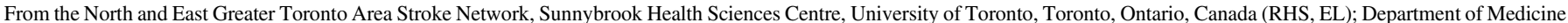

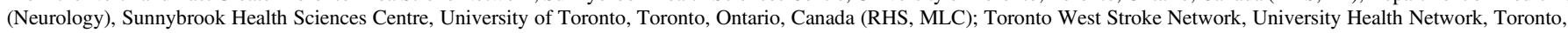

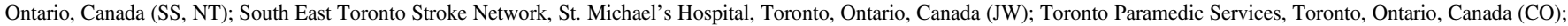

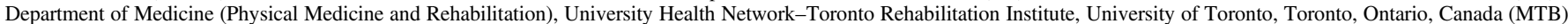
Received September 8, 2016. Final Revisions Submitted December 8, 2016. Date of Acceptance December 27, 2016.

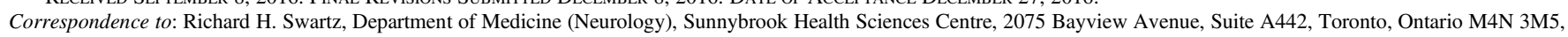

Canada. Email: rick.swartz@ sunnybrook.ca. 


\section{BACKGROUND}

Hyperacute stroke is a medical emergency for which time is of the essence. It has been estimated that every minute a stroke remains untreated results in the death of 1.9 million neurons, ${ }^{1}$ and the odds of a good outcome from treatment with tissue plasminogen activator (tPA) decline steadily over time: from 2.5 in the first 1.5 hours down to 1.25 if treated at 4.5 hours. $^{2}$ Based on a large observational prospective study, ${ }^{3}$ each minute reduction in onset-to-treatment time results in, on average, 1.8 days of extra healthy life, while a 15-minute reduction provides an average equivalent of 1 month of additional disability-free life. Around the world, a wide variety of local and national initiatives have facilitated quality improvement efforts, focusing on reducing door-to-needle treatment times, resulting in measurable changes in process with demonstrated improvements in patient outcomes. ${ }^{4-7}$ Recent data from the "Get with the Guidelines" initiative have shown significant reductions in door-to-needle treatment times that have directly translated into clinically meaningful benefits: each 15 -minute reduction in onsetto-treatment time improved survival by $4 \%$, led to a $4 \%$ greater chance of independent ambulation at discharge and a $3 \%$ greater likelihood of discharge home. ${ }^{8}$

In contrast, the current study focuses on the time "before the door," an understudied area of care that is described as the time from symptom onset to hospital presentation. An important factor with respect to time "before the door" is the decision to utilize EMS transport. It is well established that patients with stroke who use EMS are typically brought to dedicated stroke centres, and it is clear that even in those centres patients brought by EMS are evaluated and treated faster than those who do not arrive by ambulance. ${ }^{9,10}$ In regions where coordinated EMS redirection and repatriation protocols exist, they can greatly facilitate access to hyperacute interventions. ${ }^{11}$

An unintended consequence associated with greater regionalization of specialized hyperacute care is reduced access to thrombolysis for those who do not utilize EMS transport to hospital. Across the United States, more than a third of patients do not use EMS to arrive to hospital, ${ }^{9}$ and many bring themselves directly to a local hospital, unaware that this centre may not focus on stroke. In Ontario, despite universally accessible and publicly funded EMS services, only $58 \%$ of stroke patients contact EMS for transport to hospital. ${ }^{12}$ The reasons for this are many and varied, but some important examples include the inability of patients or bystanders to recognize symptoms that indicate stroke, as well as a lack of understanding by patients/families about the stroke system and how to access it. ${ }^{13,14}$ Without a coordinated system for these "walk-in" stroke patients, transport between non-stroke and stroke centres must be arranged on a case-by-case basis, resulting in delays and limiting access to hyperacute stroke interventions.

In the present study, we developed protocols to quickly identify and move patients with acute stroke symptoms from non-hyperacute hospitals to centres providing thrombolysis (tPA), launched a multi-centre, multi-disciplinary, system-based intervention to improve access to tPA, and compared transport volumes, times and tPA rates before and after the system change.

\section{Methods}

Our study was conducted as a quality-improvement initiative and met our institutional research ethics board policy for exemption from review and written consent. Toronto has 14 acute care hospitals serviced by a single paramedic organization (the Toronto Paramedic Services [TPS]), which directs stroke patients to one of three regional stroke centres using one of the first published EMS bypass protocols, in place since $2004 .{ }^{15}$ Servicing a population of 3.5 million people in the Greater Toronto Area (GTA), the TPS has approximately 750 stroke-related transports annually. Including both EMS-transported and those brought in by family or themselves, the three stroke centres administered tPA 808 times from 2009 to 2013, roughly 202 times a year (data from the Registry of the Ontario Stroke Network).

As part of a stroke system improvement initiative, a working group was established with the support of the leaders of the acute care hospitals and the TPS to develop a process to ensure access to three regional stroke centre (RSC) hyperacute stroke services from 11 non-hyperacute hospitals. The group was comprised of clinicians and administrators representing the TPS, the Toronto Stroke Networks, and emergency, general medicine and neurology from all affected hospital sites.

The working group reviewed current processes in place for medical redirection and repatriation of acute stroke patients (Supplemental Figure 1) as well as those that achieve a similar outcome for other patient populations (e.g., for ST-elevation myocardial infarcts). They collaboratively developed a Walk-In Code Stroke Protocol (Figure 1) that includes: standardized screening criteria, ${ }^{15-17}$ an EMS priority activation process with target response times, standardized communication protocols, and incident reporting mechanisms (Supplemental Figures 2 and 3). The protocol was reviewed for feasibility and consensus on implementation through consultation with all involved organizations. The working group met quarterly, with monthly updates and regular communication between members to accomplish this. The development and implementation of the protocol was grounded in knowledge-translation principles-for example, collaborative development by stakeholders, use of educational materials, monitoring and evaluation of the protocol, and provision of the opportunity to assess barriers to impletmentation. ${ }^{18}$ The protocol was specifically designed for patients with new onset of a focal neurological deficit within 3.5 hours of a clearly determined time of symptom onset or the time the patient was "last seen in a usual state of health," who are most likely to be eligible for tPA. Those with hemodynamic or respiratory instability required stabilization at the local hospital and transport by advanced paramedic EMS and were excluded from this protocol. The key components of the EMS Transport Protocol are summarized in Table 1. In addition, the working group identified the key metrics for monitoring and reporting (Table 2 ).

During protocol development, data were captured on the "pre" phase, identifying the number of cases, transport times and tPA treatment rates for patients transported during the 18 months prior to the launch of study education and roll-out. Protocols were distributed to sites during the "pre" phase to support the study launch and allow for a period of run-in before measuring their impact.

The Walk-In Code Stroke Protocol was implemented across 14 acute hospital sites within the GTA in December of 2012. Implementation of the protocol included communication with emergency department managers at all hospitals; development and distribution of standardized assessment and transfer records, guidelines and education materials for physicians, nurses and unit clerks indicating the roles and steps required to activate the protocol; and communication and education for EMS (dispatch 


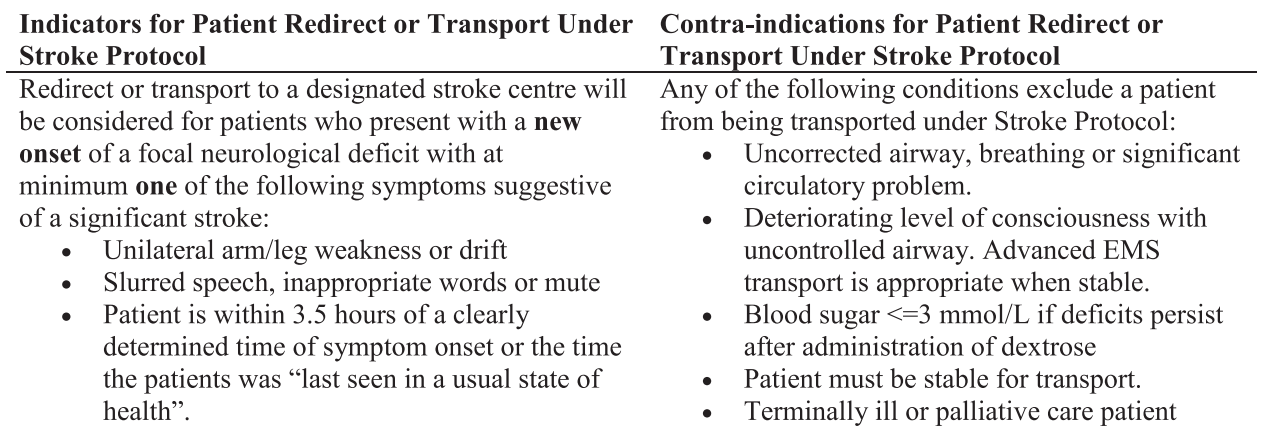

If patient arrives at non-RSC ED with hyper-acute stroke symptoms:

1. Apply stroke protocol indicators to determine if patient meets criteria for transfer to RSC

2. Contact with RSC stroke neurologist ${ }^{1}$ if diagnosis uncertain OR stroke symptoms are mild

3. Orders:

a. Large bore IV (saline)

b. Blood work (Phone receiving RSC ED nurse in charge with blood results if not available at time of transfer. Fax results when available to RSC):

i. INR, CBC, glucose, creatinine and electrolytes

c. No CT SCAN required

4. Call EMS and identify need for "Emergency Transfer Code Stroke Patient" either "Code Stroke Stable" or "Code Stroke Unstable" XXX-XXX-XXXX or use dedicated EMS phone line.

Unstable" for EMS transfer purposes means:

a. Requiring ventilatory support, or

b. Requiring inotropic support (Dopamine), or

c. Having sustained a cardiac arrest at any time in this episode of care

5. Complete Code Stroke transfer form and send with patient along with copy of patient chart

6. Pre-notification: Community hospital ED physician to contact on call stroke neurologist at RSC (if not previously contacted) to notify of Code Stroke transfer.

7. EMS will contact receiving RSC emergency department when patient on route

If patient does not meet Code Stroke protocol indicators but acute stroke is determined:

1. Determine if medically stable

2. Admit to stroke unit

a. If no stroke unit, contact Toronto EMS for non-urgent transfer to nearest appropriate hospital with an acute stroke unit (only applies to the following facilities: MSH and UHN-TGH transfer to UHNTWH, HRRH-Finch transfer to Church site, TSH-Birchmount transfer to General site)

Repatriation

Stroke patients who are medically stable and no longer require the specialized services of a Regional Stroke Centre will be repatriated to the sending hospital if it is a stroke unit hospital and if not, to the nearest appropriate stroke unit hospital. Patients who are determined to be non-stroke on arrival at RSC will be repatriated to the sending hospital. If patient has active medical issues being managed at another RSC, consideration may be given for repatriation to the alternate RSC if in the patient's best interest.

Figure 1: Walk-In Code Stroke Protocol: patient in emergency department of non-regional stroke centre.

and paramedics) and for the physicians and staff at both sending and receiving sites. Simultaneously, an addendum was added to an existing redirection and repatriation memorandum of understanding between all hospitals in the GTA (Supplemental Figure 1). The addendum reflects processes for patients walking into their local non-hyperacute hospital and subsequently being transferred to an RSC under the developed Walk-In Code Stroke Protocol, and repatriation back to the sending facility once the services of an RSC are no longer required.

System-wide communication tools were developed to report incidents (inappropriate transfers, concerns around repatriation, communication difficulties; see Supplemental Figures 2 and 3). Incidents were categorized as relevant to the walk-in protocol, EMS bypass protocol or repatriation (Figure 2) to facilitate analysis of gaps or concerns in the system. The "walk-in protocol" refers to system concerns identified in cases sent from a local hospital to a regional centre (e.g., inappropriate patient transfer).
"Bypass protocol" refers to incidents of patients' system concerns identified in cases brought directly to a regional centre by EMS. "Repatriation" refers to system concerns identified in cases sent from regional centres back to the closest hospital they were sent from or bypassed (e.g., delays in accepting the patient, incomplete information provided by the sending hospital).

This protocol leveraged existing efforts in patient transport developed for cardiac emergencies such as ST-elevation myocardial infarction. In cardiac arrest, EMS targets a pickup time of less than 9 minutes ( 8 minutes and 59 seconds or less). In order to facilitate more rapid pickup and transfer of stroke patients, this number was selected by the EMS as a target response time for stroke, despite the fact that all airway and circulatory issues were required to be stable. This target alone may have helped to reinforce the urgency of transport for paramedics. Reported incidents were addressed locally with those involved, reported immediately and monitored by the working group. 


\section{Table 1: Key components of EMS transport protocol}

\begin{tabular}{|c|c|c|}
\hline \multicolumn{2}{|l|}{ Key components (Bosk et al., 2011) ${ }^{22}$} & \multirow{2}{*}{$\begin{array}{l}\text { Toronto Stroke Network protocols and processes } \\
\text { Destination hospitals to which eligible patients will be transferred: }\end{array}$} \\
\hline $\begin{array}{l}\text { Identifying a destination / identifying } \\
\text { referral sources }\end{array}$ & $\begin{array}{l}\text { Identify regional stroke } \\
\text { centres (RSCs) }\end{array}$ & \\
\hline & & - Hospital provides 24/7 hyperacute stroke care, including laboratory, imaging and on-call stroke teams \\
\hline & & - Hospital has a designated stroke unit \\
\hline & & - Hospital has neurosurgical capacity \\
\hline & Identify referring hospitals & $\begin{array}{l}\text { Hospitals with an emergency department where patients "walk in," are brought by family or arrive by } \\
\text { EMS }\end{array}$ \\
\hline & & - Does not provide hyperacute stroke care \\
\hline & & - No stroke unit \\
\hline \multirow{5}{*}{$\begin{array}{l}\text { Identifying transfer-eligible patients / } \\
\text { negotiating the transfer }\end{array}$} & Eligibility criteria & Protocol in place that clearly articulates eligibility criteria of patients for urgent transfer to stroke centre \\
\hline & Patient transfer & Negotiation not required, standard protocol in place \\
\hline & Repatriation guidelines & $\begin{array}{l}\text { Agreement in place between all participating hospitals that clearly articulates who, when and how } \\
\text { patients will be returned to referring hospitals if: }\end{array}$ \\
\hline & & - Patient does not require hyperacute stroke care \\
\hline & & - Education information for patients to help understand why repatriation necessary \\
\hline \multirow[t]{4}{*}{ Accomplishing the transfer } & $\begin{array}{l}\text { Paramedic systems } \\
\text { engaged }\end{array}$ & Protocol in place to support urgent pick up and transfer to closest stroke centre: \\
\hline & & - Target pick up time defined \\
\hline & & - Performance monitoring in place for activation and transport times \\
\hline & & - Paramedic education \\
\hline
\end{tabular}

Data were abstracted from the EMS databases and hyperacute centre administrative databases. It was then analysed for the pre-implementation period of 18 months from June of 2011 to December of 2012, as well as for the 18 months postimplementation period from December of 2012 to June of 2014. The Shapiro-Wilk test was utilized to test whether the data were parametric (normally distributed) or non-parametric (not normally distributed). The Wilcoxon rank-sum test was used as a non-parametric alternative to the two-sample $t$ test.

\section{RESUlTS}

Comparing the 18-month blocks before and after protocol implementation, the number of walk-in patients transferred increased by $80 \%$ from 103 to 185 . The number of walk-in patients receiving tPA increased by $68 \%$ from 34 to 59 . The proportion of patients receiving tPA was not significantly different (34 vs. 31\%; Table 2). Average total transport times decreased by 17 minutes, and the median total transport time decreased from 46 to 36 minutes. The incident feedback mechanism was utilized regularly. In total, process concerns were raised at either sending or receiving hospitals in $17 \%$ of cases; however, reported incidents decreased from 23 in the first 6 months to 4 in each of the next 6-month blocks (Figure 2).

During the 18 months prior to implementation, transfers were arranged on an individual basis. The time spent at each sending hospital to find and notify an accepting physician at the receiving hospital before calling for EMS transport was not documented.

Table 2: Comparison of metrics pre- and post-protocol implementation

\begin{tabular}{|c|c|c|c|c|}
\hline & Pre-implementation ${ }^{\dagger}$ & $\begin{array}{c}\text { Post- } \\
\text { implementation }^{*}\end{array}$ & $\begin{array}{c}\text { Statistical test } \\
\text { utilized }\end{array}$ & $p$ value \\
\hline Number of walk-in patients transported & 103 & 185 & N/A & N/A \\
\hline Number of walk-in patients achieving EMS target arrival time ${ }^{*}$ at sending hospital (\%) & $35(33.98 \%)$ & $110(59.46 \%)$ & Chi-square & $<0.0001$ \\
\hline Mean response time to arrive at local hospital (mean, $S D, \min , \max )$ & $18.70 \pm 20.92(0,123)$ & $8.39 \pm 4.50(1,37)$ & Wilcoxon rank-sum & $<0.0001$ \\
\hline Time loading in hospital (mean, $S D$ ) & $16.05 \pm 9.49$ & $14.37 \pm 7.88$ & Wilcoxon rank-sum & 0.21 \\
\hline Total EMS transport time (mean, $S D$, median) & $54.56 \pm 31.19(46)$ & $37.86 \pm 13.26(36)$ & Wilcoxon rank-sum & $<0.0001$ \\
\hline Transport time to regional centre (mean, $S D$ ) & $20.01 \pm 12.09$ & $15.11 \pm 10.01$ & Wilcoxon rank-sum & 0.0004 \\
\hline Number and proportion of walk-in code stroke patients that received tPA & $34(34.09 \%)$ & $59(31.34 \%)$ & N/A & N/A \\
\hline Number and proportion of walk-in code stroke patients repatriated to the sending hospital & $7(6.79 \%)$ & $21(11.35 \%)$ & N/A & N/A \\
\hline
\end{tabular}

*8 minutes 59 seconds. ${ }^{\dagger} J u n e$ 2011-December 2012. ${ }^{*}$ December 2012-June 2014. 


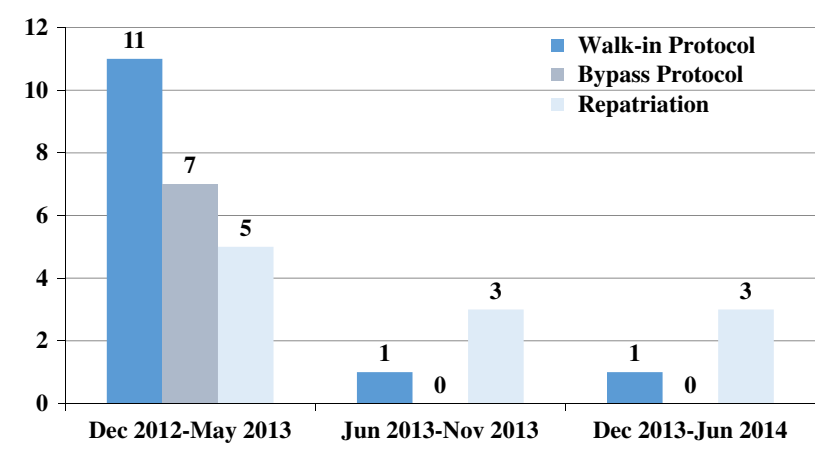

Figure 2: Incident reporting from December of 2012 to June of 2014.

Anecdotal data suggest that this was another key contributor to delays that could take anywhere from a few minutes to over an hour (Swartz RH, unpublished data, 2015). After implementation of the walk-in protocol, this step was no longer required. Thus, the total time saved was greater than that reported; however, we have no way to quantify this change.

\section{Discussion}

Numerous authors have evaluated methods to reduce in-hospital treatment times ${ }^{4,5,8}$ and to increase regionalization of stroke care. ${ }^{10,19,20}$ Some studies have even evaluated system-based interventions to improve access to tPA services and reduce times for assessment and transfer. ${ }^{1,21}$ However, to our knowledge, no study has been designed to assess a system-based intervention for "walk-in" acute stroke patients as part of a broader redirection and repatriation protocol to improve access to tPA services, and to reduce the time taken for assessment and transfer. Implementation of a system-wide process for identification, prioritization and transport of walk-in stroke patients resulted in greater numbers of patients sent to stroke centres for evaluation, more patients receiving tPA for hyperacute stroke and significant improvements in EMS response times. The ultimate goal of this process change is to facilitate stroke care and decrease onset-to-needle times for walkin patients to maximize likelihood of positive outcomes post-stroke. Our results suggest that this protocol accomplished these goals.

Based on data from the Registry of the Ontario Stroke Network, tPA is given approximately 202 times per year by the RSCs included in our study. Based on this number, we can estimate that roughly $17 \%$ of walk-in patients received tPA prior to our protocol implementation and that $29 \%$ of walk-in patients received tPA after protocol implementation. A significant increase in the percentage of walk-in patients receiving tPA can be seen. The protocol resulted in an $80 \%$ increase in transfers with no significant decline in the rates of tPA administration, suggesting that the facilitated access did not result in a large influx of inappropriate patients. While the total number of patients receiving tPA was increased with this protocol, the proportion of patients who received tPA did not change. Descriptive data suggest that the reasons for tPA not being received at RSCs are similar among walk-in patients and patients coming to hospital on existing bypass protocols (stroke mimics, patients with mild/resolved infarcts, haemorrhage and time window). While acquiring imaging before patient transfer is initiated could increase the proportion of patients treated with tPA, this would also introduce significant additional delays in transfer, especially at low-volume centres that are ill-equipped for 24/7 rapid imaging access. This is further supported by the continued low rate of repatriations (6.7 vs. $11.4 \%$ ) back to the sending hospitals, even after the protocols improved the appropriateness and ease with which patients could be repatriated. Finally, reported incidents of problematic transfers decreased as the study progressed, demonstrating the benefits of the ongoing communication protocols. In all three areas measured (walk-in, bypass and repatriation), reported incidents decreased after 6 months to a low and stable level. System-wide change requires the education and familiarity of those who use it, and this process was adopted quickly, with good success.

Our initiative of regionalization addressed four processes that were important in inter-hospital transfers of cardiac care identified by Bosk et al. ${ }^{22}$ : (1) identifying transfer-eligible patients, accomplished by use of standardized stroke recognition tools by triage nurses in the emergency department; (2) identifying a destination through delineation of geographic catchment areas for each regional stroke centre; (3) negotiating the transfer facilitated by development of standardized repatriation of patients and an incident feedback system to build trust and ensure that patients were not "dumped"; and (4) accomplishing the transfer achieved through EMS personnel adapting the existing CODE STEMI (ST-segment elevation myocardial infarction) protocol, in order to ensure ambulance arrival within 9 minutes of notification. ${ }^{22}$ A checklist was created to identify the generic steps required to create a successful EMS transfer protocol for acute stroke patients (Table 1). This checklist may assist other sites to replicate our success by meeting Bosk et al.' $\mathrm{s}^{22}$ criteria for inter-hospital transfers.

Few studies have reported the strategies used for implementation despite the advantages of regionalization and specialization for a number of time-sensitive conditions. ${ }^{19,23-25}$ Rokos et al. ${ }^{26}$ examined the impact of state legislative advocacy in regional systems of care and identified that central leadership and consensus from an independent stroke task force like our group was important to facilitate change in systems of care. McMeekin et al. ${ }^{20}$ did micro-simulation to model the impact of regionalization of stroke care showing significant improvements in quality of life with minimal investments, but they did not implement these changes. Cho et al. $^{27}$ describe the importance of designation of regional stroke centres and the use of an emergency medical information coordinating centre in Korea; however, in that study the proportion of patients accepted by RSCs decreased, possibly because these centres did not have sufficient resources to handle the increased patient volumes. There was no repatriation process described in that study, which was an important feature of our agreements and process to prevent regional centres from becoming overburdened.

Some of the key successful strategies used to facilitate our initiative are well-recognized implementation strategies ${ }^{18}$ that could be adopted in other jurisdictions. Barriers in the practice environment were addressed by engaging not only frontline providers but also all relevant organizational leaders. The protocol was familiar as it leveraged process that had been in place for myocardial infarction (the CODE STEMI protocol). The triage tool and forms were fairly simple to use. The EMS personnel were committed to timely (within 9 minutes) access to ambulances. In a non-blaming fashion, the team regularly reviewed incident reports where the protocols were not followed in order to identify 
and address problems in the flow of patients. Ongoing face-to face meetings ensured trust and ongoing network relationships.

Our protocol was designed for the stroke systems of the GTA. As more urban centres are facing centralization of stroke care, especially with endovascular thrombectomy procedures, there is an increasing need in all urban centres to ensure that people get to the right place. While the selection of hospitals and partnerships described here is unique to the GTA, the protocols, agreements and process could be used in any other urban centre where multiple hospitals serve a given population. Urban centres with ambulance redirect or bypass protocols may find this especially helpful. Within Canada, or in other nations with publicly funded healthcare access, these protocols and agreements are easier to implement than in large centres with financially competing hospitals and paramedic services. Adjustments to our protocol can be made with respect to different geographical areas and available resources in those areas. It could be argued that telemedicine technology provides access for rural or remote centres to resources and treatment that is otherwise not available. These protocols may be less relevant for the rural or remote areas of Canada, which may need to rely more heavily on such resources as telemedicine; however, it is also true that not every hospital in either urban or rural centres has access to telemedicine support, nor does every hospital have the volumes to sustain expertise in thrombolysis and efficient processes of care. As regionalization of stroke care continues to develop, there will inevitably continue to be centres without 24/7 laboratory, imaging or tPA access, and, even more, without access to endovascular therapies. The protocols outlined in our initiative can be used at both high- and low-volume centres.

A process that can transfer patients between emergency departments efficiently would not be necessary if they arrived at an RSC in the first place. Certainly, further public education is required to reduce the number of stroke patients who choose private transportation to get to a hospital. While it is ideal that patients call EMS immediately once symptoms are identified, many patients or families do not recognize stroke symptoms and the associated urgency. Further, initially mild symptoms can progress within a short time, so that a patient who arrives under their own power to a local hospital may rapidly develop worsening symptoms. Data from multiple regions show EMS utilization at less than $65 \%,^{9,12}$ suggesting that protocols to ensure that patients get to the right place in a timely manner will continue to be required even as public awareness grows. In response to this type of data and need, public education campaigns have emphasized calling 9-1-1 immediately at the onset of stroke symptoms. An example of this is the FAST campaign, launched by the Heart and Stroke Foundation of Canada (HSFC) in December of 2014, shortly after the end of our study period.

Our systems-based approach will likely become more relevant in the emerging era of hyperacute endovascular therapy. ${ }^{28-30}$ Recent trials proving the efficacy of intra-arterial stent retrieval for proximal occlusions rely on rapid advanced imaging (multiphase CTA or CT perfusion), integrated stroke teams and very fast treatment times. Every stroke centre providing tPA services will not be able to maintain 24/7 angiography access, making it even more important to have systems that can facilitate access to hyperacute stroke services.

With an additional short ambulance transfer, our protocol allows increased access to thrombolysis and thrombectomy, which are established and cost-effective treatment measures. Even if patients are ineligible for these therapies, our protocol allows greater access to expert stroke management from RSCs (haemorrhage or transient ischemic attack management, etc.), which may have some additional cost benefits. Given these complexities, detailed costbenefit analysis is warranted and may be explored in future research; however, this is beyond the scope of our current project.

Our study was limited by the fact that patients were not included in our working group. However, consideration was given to the patient experience overall through decisions made to minimize patient transfers, development of repatriation educational brochures (available in nine languages) to promote understanding of the need for repatriation, and development of transfer communication documents to be used between healthcare providers to promote seamlessness of treatment plans. Future reviews of the protocol should include patient and family advisors. Lastly, we could only analyse the effect of process changes on EMS transport times. The time spent by physicians at local hospitals to find a physician at regional stroke centres, to discuss the case and to obtain approval for transport adds significant delays. Unfortunately, this could not be reliably documented or abstracted in the pre-implementation phase. Post-implementation, this step was not required. Thus, protocol changes likely had greater impact than we were able to measure. Even without measuring this component of change, the EMS transport time changes are still clinically meaningful. From large national registries, it is apparent that an average reduction of more than 15 minutes can be expected to improve the survival and outcomes of those eligible for treatment. Our study had limited patient outcome data. We could not follow individual patients for short- or long-term health outcomes.

\section{CONCLUSIONS}

A consensus-based process developed across a complex system of care involving 14 urban healthcare organizations to regionalize hyperacute stroke care for walk-in stroke patients increased access to regional stroke centres with reduced transport time and increased tPA use. The processes, agreements and communication tools developed in this study are transferable to other regions to facilitate access to $\mathrm{tPA}$, and are becoming even more relevant in the emerging era of transporting patients for access to intra-arterial stroke interventions. Additional strategies for EMS education and engagement should be explored to determine whether times "before the door" can be further optimized.

\section{SUPPLEMENTARY MATERIAL}

To view supplementary materials for this article, please visit https://doi.org/10.1017/cjn.2017.40

\section{ACKNOWLEDGEMENTS}

The authors would like to acknowledge the efforts of the working group from all 14 hospitals and the Toronto Paramedic Services to develop and successfully implement the protocols described herein. The authors would also like to thank Anand Sinha (University of Waterloo) for his assistance in compiling this manuscript.

\section{Funding Sources}

RHS receives salary support from the HSFC New Investigator Award, the HSFC H.J. Barnett Scholarship and the Departments of Medicine (Sunnybrook Health Sciences Centre, University of 
Toronto); operating grant support from the HSFC (grant \# 000392 , 2012-2014); the Academic Health Science Centre Alternative Funding Plan Innovation Fund, and Canadian Institutes of Health Research (CIHR) grant no. 1012404 (2014-8).

\section{Disclosures}

Richard H. Swartz has the following disclosures: Heart and Stroke Foundation of Canada: grant no. 000392, grant recipient, stock owned N/A, RHS-PI; CIHR: grant recipient, grant no. 1012404, stock owned N/A, RHS-PI; HSFC New Investigator Award: employee, salary support, stock owned N/A, RHS-PI; HSFC H.J. Barnett Scholarship: employee, salary support, stock owned N/A, RHS-PI; Sunnybrook Health Sciences Centre, Department of Medicine: salary support, stock owned N/A, RHS-PI.

Elizabeth Linkewich, Shelley Sharp, Jacqueline Willems, Chris Olynyk, Nicola Tahair, Megan Cayley, and Mark Bayley do not have anything to disclose.

\section{Statement of Authorship}

RHS was responsible for the design, conceptualization and supervision of the study, obtaining funding, data collection, study coordination, analysis and interpretation of the data, drafting and revising the manuscript for intellectual content, as well as for accurate data analysis.

EL, SS, JW, CO, NT and MTB were responsible for design and conceptualization of the study, data collection, analysis and interpretation of the data, and drafting and revising the manuscript for intellectual content.

MC was responsible for data collection, analysis, and interpretation, as well as drafting and revising the manuscript for intellectual content.

\section{REFERENCES}

1. Saver JL. Time is brain—quantified. Stroke. 2006;37(1):263-6; Epub ahead of print Dec 8, 2005. http://stroke.ahajournals.org/content/ 37/1/263.long. Accessed February 18, 2017.

2. Gumbinger $\mathrm{C}$, Reuter $\mathrm{B}$, Stock $\mathrm{C}$, et al. Time to treatment with recombinant tissue plasminogen activator and outcome of stroke in clinical practice: retrospective analysis of hospital quality assurance data with comparison with results from randomised clinical trials. BMJ. 2014;348:g3429 https://www. ncbi.nlm.nih.gov/pmc/articles/PMC4039388/. Accessed February 18, 2017.

3. Meretoja A, Keshtkaran M, Saver JL, et al. Stroke thrombolysis: save a minute, save a day. Stroke. 2014;45(4):1053-8; Epub ahead of print Mar 13. http://stroke.ahajournals.org/content/45/4/1053. long. Accessed February 18, 2017.

4. Xian Y, Smith EE, Zhao X, et al. Strategies used by hospitals to improve speed of tissue-type plasminogen activator treatment in acute ischemic stroke. Stroke. 2014;45(5):1387-95; Epub ahead of print Apr 8. http://stroke.ahajournals.org/content/45/5/1387. long. Accessed February 18, 2017.

5. Fonarow GC, Smith EE, Saver JL, et al. Improving door-to-needle times in acute ischemic stroke: the design and rationale for the American Heart Association/American Stroke Association's "Target: Stroke" initiative. Stroke. 2011;42(10):2983-9; Epub ahead of print Sep 1. http://stroke.ahajournals.org/content/42/10/ 2983.long. Accessed February 18, 2017.

6. Meretoja A, Strbian D, Mustanoja S, Tatlisumak T, Lindsberg PJ, Kaste M. Reducing in-hospital delay to 20 minutes in stroke thrombolysis. Neurology. 2012;79(4):306-13; Epub ahead of print May 23.
7. Meretoja A, Weir L, Ugalde $\mathrm{M}$, et al. Helsinki model cut stroke thrombolysis delays to 25 minutes in Melbourne in only 4 months. Neurology. 2013;81(12):1071-6; Epub ahead of print Aug 14.

8. Saver JL, Fonarow GC, Smith EE, et al. Time to treatment with intravenous tissue plasminogen activator and outcome from acute ischemic stroke. JAMA. 2013;309(23):2480-8, http://jamanetwork. com/journals/jama/fullarticle/1697967 Accessed February 18, 2017.

9. Ekundayo OJ, Saver JL, Fonarow GC, et al. Patterns of emergency medical services use and its association with timely stroke treatment: findings from "Get with the Guidelines-Stroke.". Circ Cardiovasc Qual Outcomes. 2013;63:262-9; Epub ahead of print Apr 29. http://circoutcomes.ahajournals.org/content/6/3/262. long. Accessed February 18, 2017.

10. Rost NS, Smith EE, Pervez MA, Mello P, Dreyer P, Schwamm LH. Predictors of increased intravenous tissue plasminogen activator use among hospitals participating in the Massachusetts Primary Stroke Service Program. Circ Cardiovasc Qual Outcomes. 2012; 5(3):314-20; Epub ahead of print Apr 24. https://www.ncbi.nlm. nih.gov/pmc/articles/PMC3361890/. Accessed February 18, 2017.

11. Quain DA, Parsons MW, Loudfoot AR, et al. Improving access to acute stroke therapies: a controlled trial of organised pre-hospital and emergency care. Med J Aust. 2008;189(8):429-33; https:// www.mja.com.au/journal/2008/189/8/improving-access-acutestroke-therapies-controlled-trial-organised-pre-hospital. Accessed February 18, 2017.

12. Hall R, Linkewich B, Khan F, et al. Ontario Stroke Evaluation Report 2013: Spotlight on Secondary Stroke Prevention and Care. Supplement: Ontario Stroke Report Cards. Toronto: Institute for Clinical and Evaluative Sciences; 2013. http://www.ices.on.ca/ $\sim /$ media/Files/Atlases-Reports/2013/Stroke-evaluation-report/Reportcards.ashx/. Accessed February 18, 2017.

13. Menon SC, Pandey DK, Morgenstern LB. Critical factors determining access to acute stroke care. Neurology. 1998;51 (2):427-32.

14. Kongable GL. Code stroke: using tPA to prevent ischemic brain injury. Am J Nurs. 1997;97(11):16BB-HH.

15. Gladstone DJ, Rodan LH, Sahlas DJ, et al. A citywide prehospital protocol increases access to stroke thrombolysis in Toronto. Stroke. 2009;40(12):3841-4; Epub ahead of print Oct 29. http://stroke. ahajournals.org/content/40/12/3841.long. Accessed February 18, 2017.

16. Chenkin J, Gladstone DJ, Verbeek PR, et al. Predictive value of the Ontario prehospital stroke screening tool for the identification of patients with acute stroke. Prehosp Emerg Care. 2009; 13(2):153-9.

17. Verma A, Gladstone DJ, Fang J, Chenkin J, Black SE, Verbeek PR. Effect of online medical control on prehospital Code Stroke triage. CJEM. 2010;12(2):103-10.

18. Straus SE, Tetroe JM, Graham ID. Knowledge translation is the use of knowledge in health care decision making. J Clin Epidemiol. 2011;64(1):6-10; http://www.jclinepi.com/article/S0895-4356(09) 00267-4/pdf. Accessed February 18, 2017.

19. Carr BG, Matthew Edwards J, Martinez R, et al. Regionalized care for time-critical conditions: lessons learned from existing networks. Acad Emerg Med. 2010;17(12):1354-8.

20. McMeekin P, Gray J, Ford GA, Rodgers H, Price CI. Modelling the efficiency of local versus central provision of intravenous thrombolysis after acute ischemic stroke. Stroke. 2013;4411:3114-9; Epub ahead of print Aug 27. http://stroke.ahajournals.org/content/ 44/11/3114.long. Accessed February 18, 2017.

21. Berglund A, Svensson L, Sjöstrand C, et al. Higher prehospital priority level of stroke improves thrombolysis frequency and time to stroke unit: the Hyperacute Stroke Alarm (HASTA) study. Stroke. 2012;43(10):2666-70; http://stroke.ahajournals.org/ content/43/10/2666.long. Accessed February 18, 2017.

22. Bosk EA, Veinot T, Iwashyna TJ. Which patients and where: a qualitative study of patient transfers from community hospitals. Med Care. 2011;49(6):5928 https://www.ncbi.nlm.nih.gov/pmc/ articles/PMC3103266/. Accessed February 18, 2017. 
23. Edwards JM, Carr BG. Improving patient outcomes from acute cardiovascular events through regionalized systems of care. Hosp Pract (1995). 2010;38(4):54-62.

24. Higashida R, Alberts MJ, Alexander DN, et al. Interactions within stroke systems of care: a policy statement from the American Heart Association/American Stroke Association. Stroke. 2013; 44(10):2961-84; Epub ahead of print Aug 29. http://stroke. ahajournals.org/content/44/10/2961.long. Accessed February 18, 2017.

25. Jauch EC, Saver JL, Adams HP, et al. Guidelines for the early management of patients with acute ischemic stroke: a guideline for healthcare professionals from the American Heart Association/American Stroke Association. Stroke. 2013;44(3):870-947; Epub ahead of print Jan 31. http://stroke.ahajournals.org/content/ 44/3/870.long. Accessed February 18, 2017.

26. Rokos IC, Schwamm LH, Konig M, et al. Variable impact of state legislative advocacy on registry participation and regional systems of care implementation: a policy statement from the American Heart Association. Circulation. 2013;128(16): 1799-1809; Epub ahead of print Sep 16. http://circ.ahajournals. org/content/128/16/1799.long. Accessed February 18, 2017.
27. Cho SJ, Sung SM, Park SW, et al. Changes in interhospital transfer patterns of acute ischemic stroke patients in the regional stroke care system after designation of a cerebrovascular-specified center. Chonnam Med J. 2012;48(3):169-73; Epub ahead of print Dec 21. https://www.ncbi.nlm.nih.gov/pmc/articles/PMC3539098/. Accessed February 18, 2017.

28. Berkhemer OA, Fransen PSS, Beumer D, et al. A randomized trial of intraarterial treatment for acute ischemic stroke. N Engl J Med. 2014;372(1):11-20; Epub ahead of print Dec 17. http://www. nejm.org/doi/10.1056/NEJMoa1411587\#t=articleTop. Accessed February 18, 2017.

29. Goyal M, Demchuk AM, Menon BK, et al. Randomized assessment of rapid endovascular treatment of ischemic stroke. N Engl J Med. 2015;372(11):1019-30; Epub ahead of print Feb 11. http://www. nejm.org/doi/pdf/10.1056/NEJMoa1414905. Accessed February $18,2017$.

30. Campbell BC, Mitchell PJ, Kleinig TJ, et al. Endovascular therapy for ischemic stroke with perfusion-imaging selection. N Engl J Med. 2015;372(11):1009-18; Epub ahead of print Feb 11. http://www. nejm.org/doi/pdf/10.1056/NEJMoa1414792. Accessed February $18,2017$. 